EPJ Web of Conferences 37, 08002 (2012)

DOI: $10.1051 /$ epjconf/20123708002

(C) Owned by the authors, published by EDP Sciences, 2012

\title{
Recent Results of Light Hadron Spectroscopy at BESIII
}

\author{
Yutie Liang ${ }^{\mathrm{a}}$ for the BESIII Collaboration \\ II. Physikalisches Institut, Giessen University, 35392, Germany
}

\begin{abstract}
Based on the 225 million $J / \psi$ events and 106 million $\psi(3686)$ events collected with the BESIII detector at the BEPCII facility, the recent results on light hadron spectroscopy are presented, including the confirmation of $X(1835)$ and observation of two new resonances in $J / \psi \rightarrow \gamma \pi^{+} \pi^{-} \eta^{\prime}$, observation of a structure $X(1870)$ in $J / \psi \rightarrow \omega \pi^{+} \pi^{-} \eta$, and the partial wave analysis of $\psi(3686) \rightarrow p \bar{p} \pi^{0}$.
\end{abstract}

\section{Introduction}

Light hadron spectroscopy has been one major platform for probing many of the dynamical aspects of strong interaction, and provide insights into non-perturbative properties of QCD. Charmonium decay is thought to be ideal laboratory to study light hadron spectroscopy and search for new hadrons, such as glueballs and hybrids, which is one of the main physics goals of the Beijing Spectrometer (BESIII). The BESIII is a large solid-angle magnetic spectrometer, which is located at the Beijing ElectronPositron Collider (BEPCII) [1]. In 2009, 225 million $J / \psi$ events and 106 million $\psi(3686)$ event were taken. In this paper we present the progress on the light hadron spectroscopy based on these two data samples.

\section{Confirmation of $X(1835)$ and Observation of the Resonances $X(2120)$ and $X(2370)$}

$X(1835)$ was first observed in $J / \psi \rightarrow \gamma \pi^{+} \pi^{-} \eta \prime$ decays with a statistical significance of $7.7 \sigma$ by the BESII experiment [2]. Many theoretical interpretations have been raised to settle on the nature of this resonance, such as a $p \bar{p}$ bound state [3], a glueball [4], a radial excitation of the $\eta^{\prime}$ meson [5], etc.. With the accumulation of a statistically higher $J / \psi$ sample at BESIII, the confirmation of this resonance and a search for other possible resonances become possible.

In this analysis [6], $\eta^{\prime}$ is reconstructed by two decay modes, $\eta^{\prime} \rightarrow \gamma \rho$ and $\eta^{\prime} \rightarrow \pi^{+} \pi^{-} \eta$. Figure 1 shows the $\pi^{+} \pi^{-} \eta$ ' invariant mass spectrum for the combined two $\eta^{\prime}$ decay modes. In addition to $X(1835)$, one resonance $f_{1}(1510)$ at the lower mass region and two unknown structures around $2.1 \mathrm{GeV} / \mathrm{c}^{2}$ and $2.4 \mathrm{GeV} / \mathrm{c}^{2}$ are observed. Fits to the mass spectrum are also shown in Figure 1, in which the resonance is described by four efficiency-corrected Breit-Wigner functions convoluted with a Gaussian. The background is described by a non-resonant $\pi^{+} \pi^{-} \eta \prime$ contribution and other background contributions, such as the non- $\eta$ ' background.

Taking into account the systematic uncertainties, the statistical significance of the $X(1835)$ is larger than $20 \sigma$, while those for the $f_{1}(1510)$ and the two unknown structures are larger than $5.7 \sigma, 7.2 \sigma$ and $6.4 \sigma$, respectively. The mass and width of $X(1835)$ and the two unknown structures have been measured and are shown in Table 1 . According to the measured mass values, the two unknown structures are labeled as $X(2120)$ and $X(2370)$. For the $X(1835)$, the angular distribution of the radiative photon is studied and consistent with a pseudoscalar assignment.

\footnotetext{
a e-mail: yutie.liang@physik.uni-giessen.de
}

This is an Open Access article distributed under the terms of the Creative Commons Attribution License 2.0, which permits unrestricted use, distribution, and reproduction in any medium, provided the original work is properly cited. 


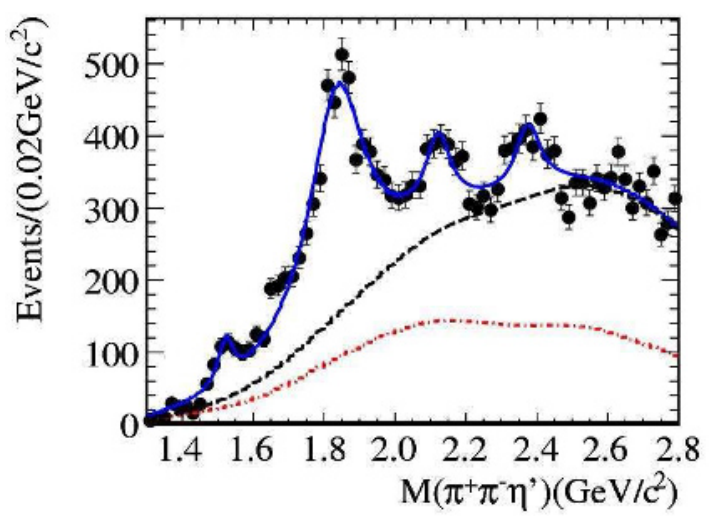

Fig. 1. The dots with error bar show the $\pi^{+} \pi^{-} \eta$ ' invariant mass distribution for the selected events from the two $\eta^{\prime}$ decay modes. Mass spectrum fitting with four resonances; here, the dashed-dotted line represents contributions of non- $\eta^{\prime}$ events and the $\pi^{0} \pi^{+} \pi^{-} \eta$ ' background for two $\eta^{\prime}$ decay modes, and the dashed line is contributions of the total background and nonresonant $\pi^{+} \pi^{-} \eta^{\prime}$ process.

Table 1. The masses and widths of $X(1835), X(2120)$ and $X(2370)$.

\begin{tabular}{cccc}
\hline Resonance & $X(1835)$ & $X(2120)$ & $X(2370)$ \\
\hline Mass $\left(\mathrm{MeV} / c^{2}\right)$ & $1836.5 \pm 3.0(\text { stat })_{-2.1}^{+5.6}($ syst $)$ & $2122.4 \pm 6.7(\text { stat })_{-2.7}^{+4.7}($ syst $)$ & $2376.3 \pm 8.7(\text { stat })_{-4.3}^{+3.2}($ syst $)$ \\
Width $\left(\mathrm{MeV} / c^{2}\right)$ & $190 \pm 9(\text { stat })_{-36}^{+38}($ syst $)$ & $83 \pm 16(\text { stat })_{-11}^{+31}($ syst $)$ & $83 \pm 17(\text { stat })_{-6}^{+44}($ syst $)$ \\
\hline
\end{tabular}

\section{Observation of a resonance $X(1870)$ in $J / \psi \rightarrow \omega \pi^{+} \pi^{-} \eta$}

Inspired by the observation of $X(1835)$ in $J / \psi \rightarrow \gamma \pi^{+} \pi^{-} \eta^{\prime}$, a study of $J / \psi$ hadronic decay, $J / \psi \rightarrow$ $\omega \pi^{+} \pi^{-} \eta$, is performed. A new structure in the $\pi^{+} \pi^{-} \eta$ invariant mass spectrum around $1.87 \mathrm{GeV} / \mathrm{c}^{2}$ [7] is observed with the requirement of $M_{\eta \pi^{+}}$or $M_{\eta \pi^{-}}$in $a_{0}(980)$ mass window, as shown in Fig.2. Three peaks are clearly seen in the spectrum. The first two are inferred to be the $f_{1}(1285)$ and $\eta(1405)$ based on the fit results. The last peak located around $1.87 \mathrm{GeV} / \mathrm{c}^{2}$ is named as $X(1870)$. In the fit of the mass spectrum the three signal peaks are parameterized by Breit-Wigner functions convoluted with a Gaussian resolution function and multiplied by an efficiency curve.

The statistical significance of the $X(1870)$ is determined to be $7.2 \sigma$, with a mass of $1877.3 \pm 6.3_{-7.4}^{+3.4}$ $\mathrm{MeV} / c^{2}$, and a width of $57 \pm 12_{-4}^{+19} \mathrm{MeV} / c^{2}$. A further study is needed to answer the question whether the $X(1870)$ and $X(1835)$ are the same resonance.

\section{A partial wave analysis of $\psi(3686) \rightarrow p \bar{p} \pi^{0}$}

The $\mathrm{N}^{*}$ study is one important branch in the BESIII physics program. Different from the traditional $\mathrm{N}^{*}$ studies using tagged photons or pion beam, a method via decays of charmonium states is applied in BESIII. One great feature of the method at BESIII is the natural isospin filter which make the analysis less complicated.

In this analysis, the final state is characterized by one proton, one antiproton and a $\pi^{0}$. The Dalitz plot of this decay is shown in Fig. 3 (a). The invariant mass of $p \bar{p}$ is displayed in Fig. 3 (b), in which a threshold enhancement and the $J / \psi$ peak are seen. To exclude events with $p \bar{p}$ arising from $J / \psi$ decay, a cut of $\left|M_{p \bar{p}}-M_{J / \psi}\right|>40 \mathrm{MeV} / c^{2}$ is applied. The survived events are shown in plots (c) and (d) of Fig. 3, displayed as the invariant mass of $p \pi^{0}$ and $\bar{p} \pi^{0}$, in which some $N^{*}$ states can been seen.

To better understand the components of this decay, a partial wave analysis taking into account the possible interferences is pursued. In the PWA, $19 N^{*}$ resonances and a hypothetical $p \bar{p}$ resonance are 


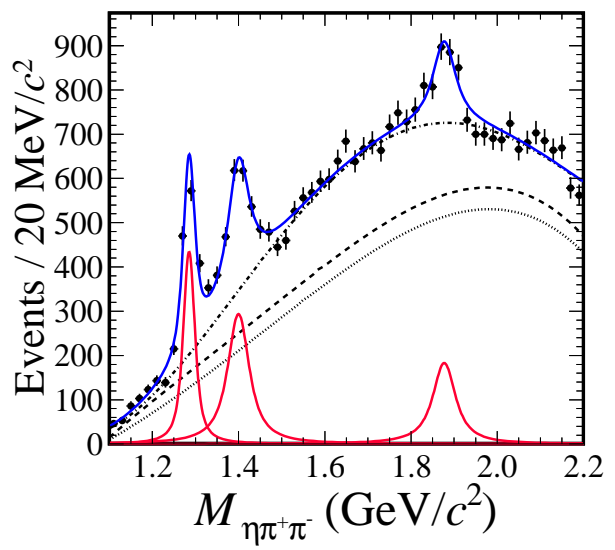

Fig. 2. Results of the fit to the $M\left(\eta \pi^{+} \pi^{-}\right)$mass distribution for events with either the $\eta \pi^{+}$or $\eta \pi^{-}$in the $a_{0}(980)$ mass window. The dotted curve shows the contribution of non- $\omega$ and/or non- $a_{0}(980)$ background, the dashed line also includes the contribution from $J / \psi \rightarrow b_{1}(1235) a_{0}(980)$, and the dot-dashed curve indicates the total background with the nonresonant $J / \psi \rightarrow \omega a_{0}^{ \pm}(980) \pi^{ \pm}$included.
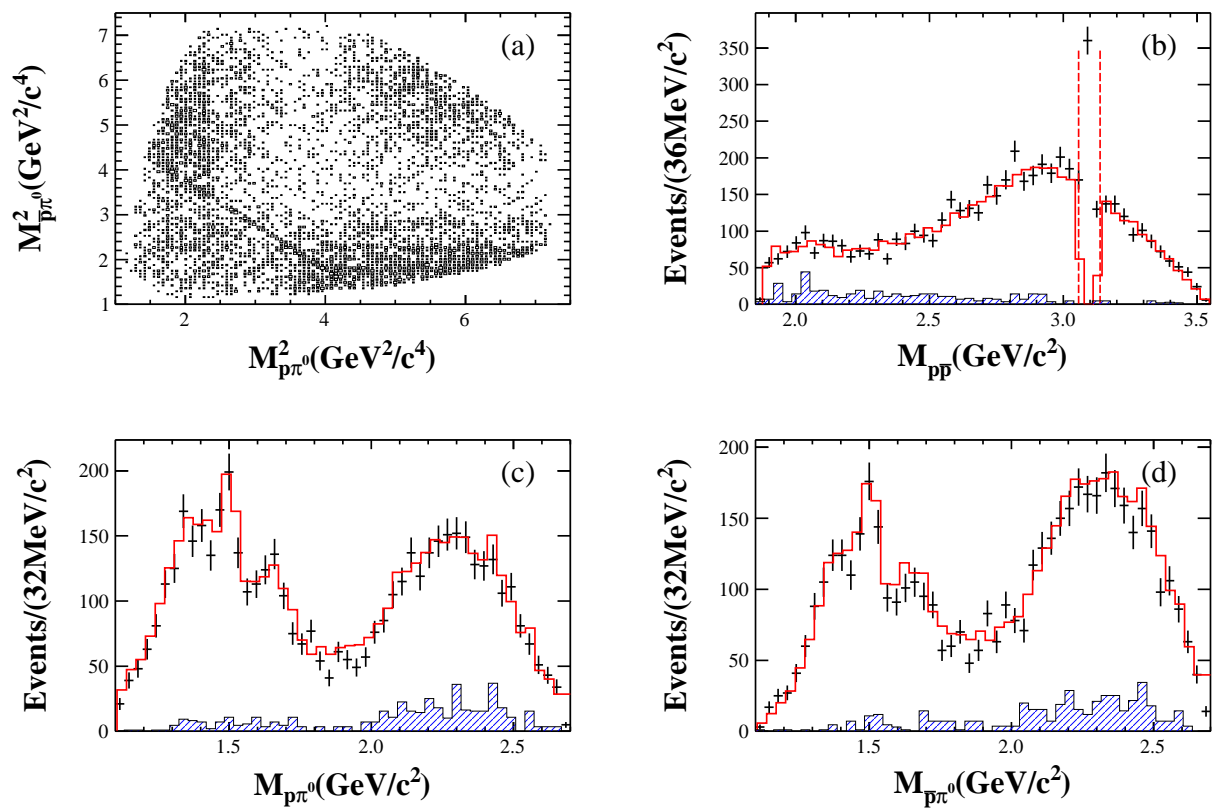

Fig. 3. (a) The Dalitz plot of $\psi(3686) \rightarrow p \bar{p} \pi^{0}$, the invariant mass spectra of (b) $p \bar{p}$, (c) $p \pi^{0}$, and (d) $\bar{p} \pi^{0}$. The dashed lines in (b) show the cut at the $J / \psi$ mass region. The crosses represent the experimental data, and the shaded histograms show the background from continuum process and $\pi^{0}$ sideband. The histograms in solid line show the sum of MC prediction and the background. 
Table 2. The optimized mass, width and significance (Sig.) of the seven significant $N^{*}$ resonances. $\Delta S$ represents the change of the log likelihood value. $\Delta N_{d o f}$ is the change of the number of free parameters in the fit. In the second and third columns, the first error is statistical and the second is systematic.

\begin{tabular}{|c|c|c|c|c|c|}
\hline Resonance & $\mathrm{M}\left(\mathrm{MeV} / c^{2}\right)$ & $\Gamma\left(\mathrm{MeV} / c^{2}\right)$ & $\Delta S$ & $\Delta N_{d o f}$ & Sig. \\
\hline$N(1440)$ & $1390_{-21-31}^{+11+21}$ & $340_{-40-156}^{+46+70}$ & 72.5 & 4 & $11.5 \sigma$ \\
\hline$N(1520)$ & $1510^{-21-30}$ & $\begin{array}{c}-40-156 \\
115^{+20+0}\end{array}$ & 19.8 & 6 & $5.0 \sigma$ \\
\hline$N(1535)$ & $1535^{-79+15}$ & $\begin{array}{l}-15-40 \\
120^{+20+0}\end{array}$ & 49.4 & 4 & $9.3 \sigma$ \\
\hline$N(1650)$ & $\begin{array}{l}-8-22 \\
1650_{-5-30}^{+5+11}\end{array}$ & $\begin{array}{l}150_{-22-50-42}^{+21+14} \\
0\end{array}$ & 82.1 & 4 & $12.2 \sigma$ \\
\hline$N(1720)$ & $1700_{-28-35}^{+30+30}$ & $\begin{array}{c}-22-50 \\
450_{-94-44}^{+109+149}\end{array}$ & 55.6 & 6 & $9.6 \sigma$ \\
\hline$N(2300)$ & $2300_{-3-2}^{+40-109}$ & $\begin{array}{l}-94-44 \\
340_{-30-58}^{+30+110}\end{array}$ & 120.7 & 4 & $15.0 \sigma$ \\
\hline$N(2570)$ & $2570_{-10-10}^{+19+34}$ & $\begin{array}{c}-30-58 \\
250_{-24-21}^{+14+69}\end{array}$ & 78.9 & 6 & $11.7 \sigma$ \\
\hline
\end{tabular}

considered. The significance of each resonance is determined. Resonances with significance greater than $5 \sigma$ are taken as significant ones and include $N(940)$ and seven $N^{*}$ resonances. The mass and width of $N^{*}$ states are varied, and the values with the best fitting result are taken as the optimized values. Table 2 lists the optimized values for the seven $N^{*}$ states. In this table, the first five $N^{*}$ resonances are consistent with the values in the Particle Data Book [8], while the last two are new. The significance of these two states are $15 \sigma$ and $11.7 \sigma$, respectively. We label these two states as $N(2300)$ and $N(2570)$, with $J^{P}$ assignment of $1 / 2^{+}$and $5 / 2^{-}$, respectively.

Using these eight significant resonances, the fit result agrees well with the data, as shown in Fig. 3 . The $\chi^{2}$ over the number of degree of freedom is 1.12 . Various checks have been performed to test the reliability of this analysis, including a spin parity check and input-output check. On the basis of the eight significant states, a scan for additional resonances has been performed with different spin parity, mass and width combinations. No extra resonance has been found to be significant. Besides the known and speculative $N^{*}$ resonances, a $1^{--} p \bar{p}$ resonance candidate described by the Breit-Wigner function has been added, as suggested by the near threshold enhancement in the $p \bar{p}$ mass distribution. The largest significance obtained is $4 \sigma$ at a mass of $2000 \mathrm{MeV} / c^{2}$ and width of $50 \mathrm{MeV} / c^{2}$, indicating that no $p \bar{p}$ resonance is required to explain the threshold enhancement.

\section{Summary}

Based on the 225 million $J / \psi$ events and 106 million $\psi(3686)$ events at BESIII, recent results on light hadron spectroscopy are reported. The $X(1835)$ is confirmed with a statistical significance of $20 \sigma$ and two other resonances $X(2120)$ and $X(2370)$ are observed. In the decay of $J / \psi \rightarrow \omega \pi^{+} \pi^{-} \eta$, a structure $X(1870)$ is observed. A PWA of $\psi(3686) \rightarrow p \bar{p} \pi^{0}$ is performed and two new $N^{*}$ resonances are reported.

\section{References}

1. M. Ablikim et al. (BES Collaboration), Nucl. Instrum. Meth. A 614, 345 (2010)

2. M. Ablikim et al. (BES Collaboration), Phys. Rev. Lett. 95, 262001 (2005)

3. A. Datta and P. J. O. Donnel, Phys. Lett. B 567, 273 (2003)

4. N. Kochelev and D. P. Min, Phys. Lett. B 633, 283-288 (2006)

5. T. Huang and S. L. Zhu, Phys. Rev. D 73, 014023 (2006)

6. M. Ablikim et al. (BES Collaboration), Phys. Rev. Lett. 106, 072002 (2011)

7. M. Ablikim et al. (BES Collaboration), Phys. Rev. Lett. 107, 182001 (2011)

8. C. Amsler et al., Phys. Lett. B 667, 1 (2008). 